\title{
CATULO, C. 16: HIS PILOSIS, UNA NUEVA INTERPRETACIÓN
}

\author{
Catullus, c. 16: his pilosis, a new interpretation
}

\author{
Marcos Carmignani* \\ UNC-CONICET \\ marcoscarmignani@unc.edu.ar
}

Palabras clave

Catulo;

carmen 16;

his pilosis;

metapoética

\section{Resumen}

En Catulo 16.10 se encuentra la expresión his pilosis, que la crítica ha vinculado, acertadamente, con lo obsceno. Este trabajo pretende sumar a esa lectura la consideración de his pilosis como una expresión metapoética, es decir, con el sentido de que los pilosi aludidos son aquellos "peludos", i.e. torpes, rústicos, incapaces de entender una poesía como la de Catulo, que se destaca por un refinado pulido de las palabras. Para ello, se analizarán cuatro elementos: 1) la interpretación general del carmen, 2) una frase de las Epistulae de Séneca que puede ser de mucha utilidad para entender nuestra propuesta, 3) la cuestión de la terminología latina vinculada con la pilosidad, y 4) la utilización que el propio Catulo realiza en otros carmina de esta terminología.

\begin{abstract}
In Catullus 16.10 the expression bis pilosis has been rightly interpreted by scholars with an obscene meaning. This paper intends to add to that reading the consideration of his pilosis as a metapoetic expression, that is, with the sense that the pilosi alluded to are those "hairy", i.e. clumsy, rustic, incapable of understanding a poetry like that of Catullus, which stands out for a refined polishing of words. To this end, four elements will be analyzed: 1) the general interpretation of the carmen, 2) a section from Seneca's Epistulae very useful to our interpretation, 3) the question of the Latin terminology linked to pilosity, and 4) the use that Catullus himself makes in other carmina of this terminology.
\end{abstract}

Keywords

Catullus; carmen 16;

bis pilosis;

metapoetics 


\section{Catulo, c. 16: his pilosis, una nueva interpretación}

Muchas veces reducido a la explícita obscenidad de su primer y último verso, que implicó la negación o el silencio acerca de su sentido, ${ }^{1}$ el c. 16 de Catulo presenta una complejidad mucho mayor de la que aparenta. El propósito de este artículo es analizar la expresión his pilosis del v. 10 desde una nueva perspectiva, hasta ahora no estudiada ni mencionada por la crítica, que, creemos, tiene mucho que ver con la poética catuliana y que puede funcionar como clave de lectura del carmen. ${ }^{2}$

Antes de comenzar el análisis, recordemos el poema:

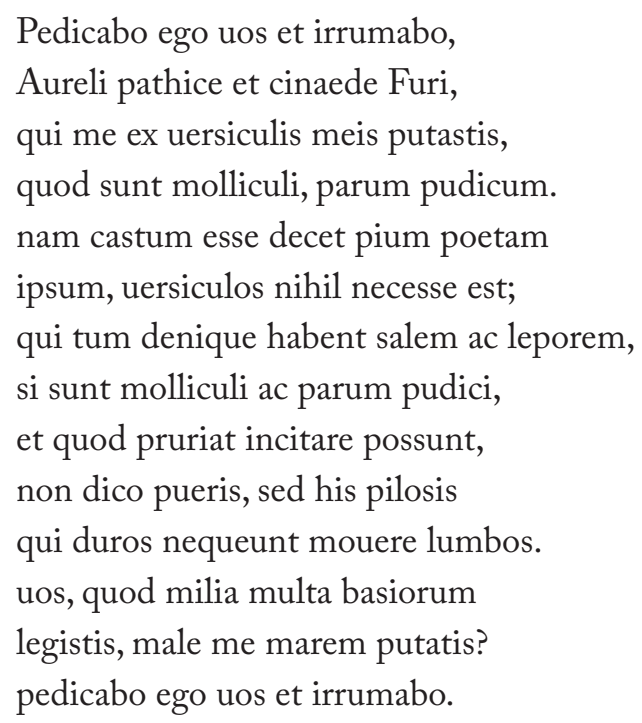

Me los voy a coger y me la van a chupar, Furio, pedazo de puto, y Aurelio, flor de maricón. Como mis versitos son apasionados, pensaron que yo era indecente. El poeta debe ser juicioso y virtuoso, pero no tienen por qué serlo sus versos. Estos resultan más picantes y sabrosos precisamente cuando son apasionados e impúdicos, porque entonces pueden incitar el ardor del deseo, no digo que en los jóvenes sin bozo, pero sí en esos viejos peludos que ya no pueden ni mover la cintura. ¿Así que ustedes leyeron 'millares de muchos besos' y pensaron que yo era menos macho? La tienen adentro, sigan mamando. ${ }^{3}$

Para anticipar el núcleo del análisis, podemos decir que, por lo general, la crítica entiende la expresión his pilosis como una referencia a la condición sexual de los involucrados, sin importar qué valoración moral se deduzca de esto. La hipótesis de lectura que proponemos es la de sumar a las interpretaciones vinculadas con lo obsceno -que, sin duda, tienen su propio peso dentro del poema- la consideración del término como una expresión metapoética, es decir, con el sentido de que los pilosi

1. Paradigmático el caso de Fordyce (1961, p. v), quien, en su comentario catuliano, revela que "a few poems which do not lend themselves to comment in English have been omitted”. El c. 16 es uno de ellos.

2. La bibliografía sobre el poema es amplísima, aunque relativamente pocos estudios consideran el problema puntual de his pilosis. En la primera parte del artículo se mencionarán aquellos análisis y comentarios que creemos fueron fundamentales para la interpretación, desde diversas perspectivas, del carmen.

3. Trad. de Marrón (2012). El texto latino es de Mynors (1958). 
aludidos son aquellos "peludos", i.e. torpes, rústicos, incapaces de entender una poesía como la de Catulo, que se destaca por ser un verdadero artifex del pulido de las palabras. Siempre en este sentido metaliterario, la "pilosidad" también podría leerse como algo que debe ser "pulido" = "afeitado", algo que evidentemente necesita un pumex, una lima o, más bien, ahora, una novacula.

Al menos cuatro elementos son necesarios para poder justificar esta hipótesis de lectura. En primer lugar, la interpretación general del carmen, que, como se dijo, implica numerosas cuestiones que la crítica ha ido proponiendo a lo largo de los años. En segundo lugar, una frase de las Epistulae de Séneca, que puede ser de mucha utilidad para entender nuestra propuesta. En tercer lugar, la cuestión de la propia terminología latina implicada o, para decirlo de otro modo, qué implicancias tiene dentro del léxico latino y, por lo tanto, de su cultura el campo semántico vinculado con la pilosidad, el vello hirsuto, etc. Por último, la utilización que el propio Catulo realiza en otros carmina de esta terminología. A partir del análisis de estos cuatro elementos -no necesariamente simétricos en extensión entre sí-, quizá podamos aportar un panorama más claro a nuestra lectura.

\section{Las interpretaciones del carmen}

Richlin (2007, p. 286, n. 10) resume claramente los principales problemas interpretativos del poema:

(a) the 'reality' of the threat in 1 and 14; (b) the nature of the situation that prompted Catullus to reply to Furius and Aurelius; (c) the identity of the versiculi $(3,6)$; (d) the meaning of molliculi $(4,8)$, parum pudicum $(4,8)$, castum (5), non dico (10), his pilosis (10), qui ... lumbos (11), male ... marem (13); (e) the referent of milia multa basiorum ('many thousands of kisses') - Cat. 5, 7, or 48, or all three?

A partir de un recorrido cronológico no exhaustivo que inicia en la segunda mitad del siglo XX -cuando el poema empieza a ser analizado con mayor atención y detenimiento, en un proceso lento pero sostenido de abandono de los condicionamientos morales-, intentaremos resumir las diferentes respuestas que la filología dio de estos problemas, pero sobre todo con el foco puesto en la cuestión de bis pilosis. Así, Kinsey (1966) tomaba esta afirmación de Kroll (1923, ad loc.): "Junge Leute sind leicht erregbar; Catull erklart es in Scherz fur eine Aufgabe der Dichtung, ältere und schon unempfindlich Männer in Aufregung zu versetzen", para acordar con él, sobre todo porque los "duri lumbi are the opposite of the soluti lumbi of Petr., Satyr., 140", pero para aclarar que Catulo afirma que sus versos no excitarán a los pueri sino solo a los pilosi, ya que non dico significa claramente "not' not 'not only" (Kinsey, 1966, p. 104). Y añade la relación con el c. 50 porque prueba que Catulo escribió poemas en el papel de los pathici y porque muestra la delgada línea que divide "affectation and real emotion": "Catullus is smitten by the lepos of Calvus just as the pilosi of our poem are excited by Catullus' salem ac leporem" (Kinsey, 1966, p. 106).

MacLeod (1973) afirma que Catulo realiza una apología de su poesía y de la potencia de sus versos, que incluso pueden excitar a los viejos, en una caracterización descarnada de la “erotic poet's function as an instructor in love", de la que surge un "paradoxical play on words, the 'soft' verses getting the better of 'stiff' or 'hard' loins. Set in these surroundings lines 5-6 are designedly incongruous" 
(MacLeod, 1973, p. 301), dado que su presentación como poeta de las Musas, para MacLeod claramente inapropiada, da paso a un ataque de cólera fingida cuyo único objetivo es reivindicar no su castidad sino sus propios poderes sexuales y los de sus versos. Por esto, "the poem is meant, as any poem is, to display the poet's characteristic qualities" (MacLeod, 1973, p. 301), y sería un error tomar en serio las críticas de Furio y Aurelio o la apología a la vida pura de Catulo: son el sustrato para poder entender la comicidad del poema.

En su artículo de 1976, Buchheit menciona que Catulo solo escribió un poema que tiene por único tema la crítica literaria: el c. 95, sobre la Zmyrna de Cinna. Buchheit argumenta que el poema es una suerte de alegoría literaria en donde los elementos sexuales representan diversos modos de escribir y leer poesía. Esto es fundamental para nuestra hipótesis.

En 1977, Lateiner publicó un estudio donde afirma que el c. 16 es un poema programático, sobre todo con su declaración de los versos 5-6. Los términos pius, castus y pudicus parecen fuera de lugar en un poema que se abre y cierra con dos de los versos más obscenos de toda la literatura clásica: justamente, esos versos son la prueba más evidente de que un poeta puede escribir versos indecentes sin serlo él mismo: "the verses which frame the poem are an example of the poem's message" (Lateiner, 1977, p. 16). Esos versos, más que una amenaza que nunca se realizará, implican un insulto para el futuro, porque, cada vez que un lector vea estas líneas, Aurelio y Furio serán realmente agredidos. Para Lateiner, la grosería de Catulo tiene que ver con pagar con la misma moneda la ofensa sufrida: sus versos fueron calificados como afeminados, de ahí el terrible insulto que invierte la situación. Sostiene que es fundamental que estos versos, calificados como molliculi y parum pudici, que pueden ser traducidos como "sensuales" y "explícitos", siempre serán una delicia poética mientras tengan salem et leporem: "Obscenity is an outlet for aggression. Obscenity in Catullus is never simply vulgarity; no poet has complained more bitterly of poor taste in life and verse” (Lateiner, 1977, p. 17). Luego, el autor nota algo interesante al decir que no puede explicar la expresión et quod pruriat incitare possunt, dado que el corpus catuliano, "however obscene it is, is not prurient, not pornographic", por lo que, en su furia, Catulo quizá "generalizes his defence of poetry. If he writes for pilosis / qui duros nequeunt movere lumbos, he claims prurience, but the extant Catullus writes for adults interested in poetry, not children or old men looking for sexual thrills" (Lateiner, 1977, p. 29, n. 7). Pero si los versiculi de Calvo excitaban a Catulo, como se lee en el c. 50.7-15, es probable que sus nugae tuvieran el mismo efecto en otros.

En su comentario, Della Corte (1977) encuadra este carmen dentro de una situación retórica definida por la Rhetorica ad Herennium, donde se describen las acciones del acusador y del acusado, quien supuestamente tuvo la voluntad de satisfacer una pasión amorosa. Así, tanto Furio y Aurelio (acusadores) como Catulo (acusado) responderían a estos principios retóricos (como, por ejemplo, el de Rhet. ad Her. 2.5: defensor primum demonstrabit vitam integram, si poterit, lo que Catulo hace declarándose castum en el v. 5). Más allá de esto, Della Corte comenta en el v. 10 que pilosis se ubica en oposición a los “levia membra dei glabri (cfr. 33.7, 61.142)" (Della Corte, 1977, ad loc.), repitiendo el mismo comentario que Lenchantin de Gubernatis (1932, ad loc.). 
Selden (1992), ${ }^{4}$ en un extenso artículo, sostiene que en el c. 16 no solo se confirma la oposición entre lenguaje performativo y lenguaje constativo (según la célebre distinción de Austin), sino que "designates their mutual resistance as the generative principle of his work" (Selden, 2007, p. 527). De este modo, la primera parte del poema toma la dimensión constativa del discurso del poeta y niega su valor heurístico. La queja del poeta sobre sus lectores literales (me ex versiculis meis putastis, / quod sunt molliculi, parum pudicum) "implicitly repudiates authenticity or candor as criteria appropriate to his reception and thereby situates the achievement of his writing outside the pale of knowledge" (Selden, 2007, p. 527). En contra de esto, Catulo afirma el poder performativo en la segunda sección, cambiando la atención crítica de lo que su obra revela a lo que su obra hace. Así, lo importante es "the extent to which his poetry operates as a captation, a seductive force or lure that emerges in the act of reading”(Selden, 2007, p. 528). Su conclusión sobre el poema es que se trata de un epítome de toda la producción catuliana, ya que "Catullus' poetry is set up both to exploit and to expose this friction (lo constativo vs lo performativo) and, to the extent that the two features of the text prove incompatible, they cannot help but rupture the appreciation of his work" (Selden, 2007, p. 538). En cuanto a bis pilosis, Selden (2007, p. 536, n. 145) cree que si los jóvenes pueden ser un objetivo justo para la dominación socio-sexual, los hombres maduros (pilosi, en marcado contrate con la típica suavidad "afeminada" de los chicos), particularmente los ciudadanos, "were theoretically off limits".

Para Wray (2001), el c. 16 es otro ejemplo de Catulo como un nuevo Arquíloco: sus endecasílabos falecios "are 'ravaging' and indeed 'raping"' yambos (Wray, 2001, p. 185). Es probable que con estos versos Catulo haya querido presentarse como el ejemplo más conspicuo conocido en la antigüedad de un poeta "santo" que escribió poemas sucios. Como explica Manwell (2007, p. 121 y ss.), Wray intenta desplazar nuestra atención hacia un poeta poco sentimental y raramente sincero, cuya principal preocupación es su estatus entre sus pares masculinos. Utilizando un modelo antropológico (basado en el de Herzfeld, 1985), Wray busca en los poemas lo que puede decirnos del otro Catulo, el vir durus, que se deleita en superar a sus amigos y enemigos por igual. Según Manwell, a Wray le debemos quizás la explicación más completa hasta la fecha de cómo las teorías románticas y modernistas de la recepción poética influyen en nuestra lectura del corpus catuliano. Aunque se quiera evitar caer en la falacia biográfica, la mayoría de los lectores de Catulo sigue pensando que el poeta nos enseña su vida y que la expresión de sus emociones es totalmente sincera. El problema surge cuando los datos que nos ofrecen los poemas de Catulo no concuerdan con la persona del amante sincero. Para responder esto, Wray propone una poética posmoderna para Catulo, donde "posmoderno" se define cuidadosamente: "a preference for the performative and ludic over the sincere and introspective; for emotional volatility over emotional intensity; for erudition, verbal wit, invention and allusivity over immediacy and 'originality'; for encyclopedic collage over meditative lyric" (Wray, 2001, p. 39). Se trata de un nuevo formalismo. Así, lo que podríamos leer como una disonancia (por ejemplo, la introducción de una alusión juguetona o la corrección de un error de un poeta anterior en un lamento) es, en realidad, un sistema diferente de valoración y comportamiento apropiado.

Por su parte, Skinner (2003) piensa que el c. 16 "dramatically sexualizes the relationship between the poet and his readers, both 'unacceptable' (Furius and Aurelius) and 'acceptable' (bis pilosis,

4. Cit. desde la versión reimpresa en Gaisser (2007). 
aroused despite their duros . . lumbos, 10-11)” (p. 225, n. 18). A pesar de lo que piensa Fitzgerald (1995, p. 49-51) acerca de que el lector se ve en una posición sexual pasiva, los sentimientos evocados por el poema pueden incluir también el deseo por su creador. Así, el lector asumiría el papel "activo" de amator. Skinner afirma que quizá lo más exacto sea decir que tanto el poeta como el público oscilan entre una posición "sexual" dominante y otra sumisa durante el proceso de lectura. Asimismo, la negación inequívoca de Catulo de escribir para enardecer a los muchachos (non dico pueris, v. 10) descarta el modelo "pederástico" que se identifica como uno de los paradigmas estándar de la lectura en el pensamiento griego clásico.

Richlin (2007) afirma con respecto al problema de his pilosis que, por muy tentador que sea comparar los vv. 10-11 con Persio 1.19-21 (como hace Buchheit, 1977, p. 342-346), "it is not possible to take the pilosis ('hairy men') as anally penetrated by the versiculi". Si bien es cierto que los poemas actúan como "aggressive phalli" en otros lugares, Catulo está diciendo que sus versiculi tienen ingenio solo si pueden despertar quod prurit ... his pilosis ... qui duros nequeunt movere lumbos. Los versiculi se describen como cinaedi, molliculi ac parum pudici; quod prurit debería ser activo y no pasivo, por analogía con c. 88.1-2, mientras que bis pilosis se contrapone a pueris y, en su contexto (vv. 8-9), debe representar a sujetos difíciles de excitar; así, qui ... lumbos es epexegético de quod ... possunt, es decir, "aunque no puedan...", mientras que duros ... lumbos es un atributo de los hombres adultos (cfr. Juv. 6.377). Por último, continúa Richlin, aunque movere lumbos puede significar movere nates ("mover las nalgas"), es difícil tomar duros movere lumbos ("mover una ingle dura”) como movere nates (2007, p. 287, n. 10).

Según Batstone (2007), es evidente que Catulo habla de su poesía y de cómo leerla. Se ha intentado negar el carácter programático de este poema declarando que no se trata de la poesía en general, sino de los "poemas de los besos" de Catulo. Tal afirmación no es un hecho, sino simplemente una decisión: la de no leer el poema de forma programática. Para Batstone, el argumento fuerte de Catulo es que la buena poesía erótica hace que incluso los viejos tiesos, que no podrían tener sexo aunque quisieran, se exciten. El carmen, entonces, no trata de lo que excita a Catulo, sino de lo que excita al lector, y Furio y Aurelio han sido malos lectores tanto en sus conclusiones como en la aceptación sexualmente pasiva de las palabras de Catulo. Así, el poema afirma que los lectores necesitan sofisticación, mientras que da la vuelta a la tortilla a sus (poco sofisticados) lectores y revela la competitividad social a la que ni siquiera la poesía puede escapar. Batstone no cree que las amenazas sean verdaderas, sino que son parte de su máscara poética.

En un denso artículo, Krostenko (2007) afirma que el c. 16 es una respuesta inteligente a quienes impugnaron la hombría de Catulo: su argumento confunde a estos lectores y su forma los atrapa. Catulo afirma que el autor y el texto son distintos (vv. 5-6) y que el verso impúdico es defendible porque su encanto es especialmente potente (vv. 7-11). Esto solo es posible gracias al lepos: sus versiculi son ingeniosos ( $\mathrm{sal}$ ), suaves (molliculi) y eróticos (parum pudici), con el suficiente poder como para excitar a los hombres mayores, físicamente ya rígidos. Para Krostenko (2007, p. 227), "the parallel with political aestheticism is striking”, ya que, como Catulo, Julio César se mostraba delicado, con el cuerpo depilado y la túnica suelta, y, como Catulo, el César fue acusado de falta de castidad, seguramente en parte debido a ese estilo: nunca se libró del rumor de que se había sometido al rey bitinio Nicomedes 
(Suet. Iul. 2.49). Y, como Catulo, el César presumía de una masculinidad extravagante. En Roma, había más de una forma de metaforizar las relaciones de poder a través del género. Para Krostenko, entonces, el esteticismo político da verdadera fuerza al argumento del poema. La defensa que hace Catulo de sí mismo implica un principio: los significados de las palabras no son absolutos, sino que dependen del marco conceptual en el que se lean. Esa posibilidad se explota en la trama del propio poema. Así, podemos analizar el lenguaje de la acusación de Furio y Aurelio: Catulo era parum pudicus y sus versos molliculi, algo bastante evidente tomado literalmente; sin embargo, molliculi suena como un término de crítica literaria, prácticamente un término de la estética neotérica propiamente dicha. Así, las críticas de Furio y Aurelio son reapropiadas como lenguaje metaliterario. Catulo también reconfigura el lenguaje moral. La imputación de mollitia e impudicitia lo lleva a afirmar que el pius poeta es castus: sin embargo, el poema reduce rápidamente la moral a las relaciones de poder, un poder sexualizado. El poeta, antes controlado, ahora controla a sus lectores. De nuevo, entonces, el parecido con el esteticismo político. Para Krostenko, la elección de decet en el v. 5 (y no de oportet o necesse est) resume perfectamente la cuestión. La palabra se encuentra en la intersección de la fuerza moral con el gusto y la apariencia: ¿significa "moralmente obligatorio", subrayando la realidad, o "de buen gusto", subrayando la apariencia? Estas duplicidades del lenguaje son trampas: atraen a los lectores con un significado y luego les arrojan otro. Así ocurre con el verso que comienza y termina el poema, una amenaza de violencia sexual degradante. Krostenko (2007, p. 227) sostiene que la primera vez el verso suena simplemente como una maldición en una disputa acalorada; en cambio, el clima del poema es diferente al final: el supuesto afeminamiento de Catulo revela un arte excitante que le permite controlar a sus lectores, y el control hace a un hombre de verdad. En este nuevo contexto, el verso inicial suena muy diferente: "the effect is rather as if a poem began 'Go to hell!' and then, having established the unrepentant sinfulness of the addressee, ended, 'And on the last day you/Go to hell"'. Krostenko (2007, p. 227) cree así que los mil besos de Catulo no deben tomarse literalmente, pero sí sus amenazas sexuales: Furio y Aurelio "took Catullus as a puer lepidus ac delicatus, as happy to be loved as to love; in Catullus' mind that was a theft of his words, and he responds to the theft like Priapus would: jumping on both their heads".

Dejamos para el final de esta sección -y rompiendo la cronología- el análisis, a nuestro juicio, más completo y agudo, realizado por Schievenin (2000), a quien seguimos en la interpretación del carmen. ${ }^{5} \mathrm{El}$ autor afirma que en el poema "una velata combinazione di reticenza, ambiguità ed eufemismo si manifesta attraverso l'intreccio, insolito, del linguaggio della sessualità con la lingua della poesia" (Schievenin, 2000, p. 195). Hay un consenso crítico general de que Catulo es un pathicus para Furio y Aurelio: esto se debe a que el poeta se representa en otros poemas de un modo sexualmente pasivo, no solo porque no avanza hacia el coito sino porque, como atestigua el famoso carmen 5, es Catulo quien pide ser besado en lugar de mostrarse en un papel activo (v. 7, da mi basia mille). Como se sabe, la acusación de pasividad sexual era una ofensa muy grave para un ciudadano romano. ${ }^{6}$ Sin embargo, Schievenin (2000, p. 198) rápidamente aporta la primera duda: "è mai possibile che Aurelio e Furio rivolgessero seriamente una tale accusa a Catullo, convinti di poterla realmente provare con

5. Extrañamente, este excelente artículo no aparece citado en el Companion de Skinner (2007).

6. Cfr. Cantarella (1988, p. 129 y ss.) y, para Catulo, Arkins (1982). Ambos textos, cit. en Schievenin (2000, p. 198, n. 13). 
i versi stessi del poeta?". Si Furio y Aurelio pensaban que estaban haciendo un chiste, la respuesta de Catulo demuestra la gravedad del asunto y la seriedad con que recibió la ofensa. El poeta ataca la falacia biográfica con un adagio propio de la literatura clásica: no se puede identificar la vida de un poeta con sus versos. La defensa de Catulo es firme y convincente porque está convencido de que sus versos tienen salem ac leporem, son molliculi ac parum pudici, $\mathrm{y}$, justamente por esto, tienen la capacidad de estimular el deseo sexual a los pilosi, incapaces de mover sus lumbos (y, por lo tanto, de tener sexo). Schievenin (2000, p. 201) agrega algo fundamental: la descripción de la potencialidad de los versos catulianos "si rivela subito già in atto: his la circoscrive, inaspettatamente, alla situazione contingente". Es decir, probar que sus versos surten efecto no es necesario porque ese deíctico bis es la prueba de que los pilosi están ahí, frente a sus ojos y a los de Furio y Aurelio. Sin embargo, continúa prudentemente Schievenin, no podemos saber a ciencia cierta quiénes son estos pilosi y qué función cumplen: "sono il vero nodo esegetico del carme” (2000, p. 201). Está claro que his pilosis aparece en oposición a pueris, en una diferenciación que sirve para aclarar el destinatario de los versos catulianos: no se habla de su efectividad en los jóvenes sino en los adultos, específicamente en aquellos que no pueden movere lumbos. Esto ha llevado a muchos críticos a identificar a estos adultos con los pathici o, más arriesgadamente, con Furio y Aurelio. ${ }^{7}$ Sin embargo, habría una clara contradicción, ya que Furio y Aurelio (o los destinatarios que fueren) nunca pueden ser pathici y pilosi al mismo tiempo: el pathicus siempre está depilado. ${ }^{8}$ Por lo tanto, la conclusión de Schievenin es que el deíctico bi devela que los pilosi no son otros que Furio y Aurelio, con una aclaración importante: "Riferito a persone, il termine pilosi rivela una sua componente di rozzezza e di sciatteria, una connotazione complessivamente negativa" (2000, p. 202) que se reafirma en la incapacidad de estos por tener sexo. El carmen es una respuesta dura y corrosiva a Furio y Aurelio, quienes se han burlado de Catulo acusándolo de patbicus, pero Catulo, que los conoce, los ataca enfatizando que la única forma de que estos pilosi puedan excitarse es leyendo sus versos eróticos. Otra pista que nos regala Schievenin (2000, p. 203) es que, si bien los destinatarios son Furio y Aurelio, también lo es "il lettore colto, destinatario finale di ogni poesia". Schievenin también explica que la autodefinición de Catulo como pius y castus le permite introducir términos de amenaza tan violentos y obscenos como pedicare et irrumare, que, por otra parte, representan probablemente el punto más bajo en el que puede caer un civis romano desde el punto de vista de la degradación sexual. Solo un poeta pius et castus tiene derecho a decir cualquier cosa, porque su poesía no tiene nada que ver con su vida. Como bien señala Schievenin, Catulo percibe el error del ataque de sus adversarios, que apuntan contra su persona, cuando en realidad son sus versos los parum pudici: de su persona poco y nada pueden decir. Por eso la importancia de his pilosis, del deíctico y de su concepción poética de una poesía performativa, porque tiene un efecto contundente en sus lectores "adultos", identificados con sus dos adversarios. Para Schievenin (2000, p. 208), pilosis asume un valor agregado cuando se lo articula con pueris:

pilosus ricorda, deteriorandolo, il valore di barbatus, termine che indica, in particolare nel linguaggio eufemistico dell'eros, l'adulto che assolve ancora a quelle funzioni passive che la sua età non

7. Schievenin (2000, p. 202, n. 21) cita como partidarios de esta postura a Baehrens (1885, p. 143-144), Richardson (1963, p. 100), Sandy (1971, p. 52), Rankin (1976, p. 91-92) y Buchheit (1976, p. 344).

8. "In ogni caso sarebbe palesemente illogico, anzi controproducente, che Catullo, nell'assunto di dimostrare che egli non è pathicus, affermasse che i suoi versi fanno eccitare proprio i pathici", cfr. Schievenin (2000, p. 202). 
richiederebbe più. Aurelio e Furio sono dunque designati con un termine che, stretto tra puer e l'omologo barbatus, finisce per ricordare da una parte la loro provocazione attiva ma dall'altra anche l'esito passivo (pathice ... cinaede) minacciato all'inizio da Catullo (pedicabo ... irrumabo).

Así, para Schievenin (2000, p. 209), pedicabo e irrumabo no tienen que ver con una reafirmación de la sexualidad de Catulo, no se trata de una reivindicación en este sentido, sino más bien es la áspera ratificación de sí mismo como poeta y de su propia poesía: "la stessa invettiva, aspra e tagliente, rappresenta il primato della parola colta e della poetica arguta e raffinata: Catullo sa elevare a dignità letteraria anche il turpiloquio da trivio".

Finalmente, y antes de pasar a nuestro segundo punto de análisis, queremos enfatizar la importancia de los vv. 5-6 para entender todo el carmen (y también la poética de Catulo). Estos versos son un manifiesto explícito de que cada escritor es libre de escribir lo que quiera sin riesgo de que sus versos fueran asociados a su modo de vida: es un manifiesto contra la "falacia biográfica". De hecho, Ovidio en sus Tristia (2.353-354) y Marcial (1.36.10-11) fueron claramente influidos por esta idea catuliana, y Plinio el Joven (Ep. 4.14) y Apuleyo (Apol. 11.3) citan los versos de Catulo como una ley. Aun Adriano dedica a Voconio este verso: lascivus versu, mente pudicus eras (carmen 2). Además, los términos pius y castus han sido históricamente mal interpretados: en realidad, pius es un epíteto que se da el propio Catulo con el sentido de "respetuoso de las Musas", ya que él, como poeta, es su sacerdote: por lo tanto, pius es sinónimo de buen poeta. Por último, queda clara la idea de que todo vale sexualmente para un hombre mientras mantenga un papel activo: la insistencia de Catulo, con la ringcomposition, también apunta a esto. Su vida sexual no tiene nada que ver con la de un pathicus.

\section{Séneca y el estilo literario: la "cosmética capilar" como pista metaliteraria}

Como afirmamos supra, nuestra lectura de pilosis se basa en una interpretación metaliteraria del término. ${ }^{9}$ Como es bien sabido, los versos de apertura del corpus catuliano ya enuncian una declaración programática: 1.1-2 Cui dono lepidum nouum libellum / arida modo pumice expolitum? (“A quién voy a dedicar mi encantador librito nuevo, recién pulido con árida piedra pómez?”). ${ }^{10} \mathrm{El}$ pumex se utilizaba para pulir los bordes del volumen pero, obviamente, a nivel metaliterario es un manifiesto poético: Catulo publicó su libro luego de haber pulido y limado el estilo, al modo de los poetae novi. Por eso su libellus es lepidus: este adjetivo, junto con facetus, "refinado, elegante, gracioso, agudo", y venustus, "agradable, refinado, encantador", entre otros, vincula su poética con un estilo pulido, erudito, lleno de gracia y siempre alejado de lo ampuloso, como puede inferirse del término libellus. La lírica de Catulo puede tratar de la vida cotidiana con un lenguaje común, pero merece la más seria de las críticas literarias. Todos estos términos y este tipo de poesía difícilmente puedan tener alguna relación con algo que sea pilosus, siempre que también entendamos esto último de manera metapoética.

9. Para cuestiones programáticas y metapoéticas en Catulo, cfr. Batstone (1998) y (2007), con bibliografía.

10. Desde acá, todas las traducciones son nuestras, salvo que se indique lo contrario. 
De este modo, dentro de estas referencias veladas a lo literario encontramos la expresión pilosis. Sin embargo, Catulo no es el único poeta latino que utilizaría una alusión a la pilosidad como referencia metapoética. De hecho, casi un siglo después, el c. 16 encuentra, según nuestra lectura, un eco en una expresión senecana que sirvió como punto de partida e inspiración para nuestra interpretación. En la Epistula 115, Séneca da indicios de que la propia literatura puede ser definida mediante términos metafóricos, en este caso, del campo de la "cosmética capilar", dando lugar a que algunos términos relacionados con la pilosidad puedan ser interpretados metapoéticamente:

Sen., Ep. 115.2: Nosti comptulos iuvenes, barba et coma nitidos, de capsula totos: nibil ab illis speraveris forte, nibil solidum. Oratio cultus animi est: si circumtonsa est et fucata et manu facta, ostendit illum quoque non esse sincerum et habere aliquid fracti. Non est ornamentum virile concinnitas. ${ }^{11}$

Así, comptulus ("elegantly dressed" traduce el $O L D$ ) es un término que el filósofo utiliza con un valor negativo, vinculado con la luxuria tan furiosamente criticada por la moralidad romana. Estos jóvenes tienen una cabellera y una barba, diríamos hoy, típica de un "metrosexual", extremadamente cuidada para un varón. De estos jóvenes, fulmina Séneca, no se puede esperar nada bueno. Luego, comienza la analogía metaliteraria; lo mismo ocurre con el estilo, que es el cultus animi, "el adorno del alma" (aunque cultus también significa "stylistic elegance, polish", cfr. OLD, s.v. 7): si es circumtonsus, debemos sospechar, porque un estilo de ese modo refleja un alma semejante. Precisamente es el término circumtonsus lo que nos importa: significa "having the hair cut or trimmed all around" (cfr. OLD), un evidente derivado de circum+tondere, raíz que será importante en los testimonios que analizaremos más adelante. La traducción que citamos, "pulido", expresa bastante bien el sentido del adjetivo y nos deja metidos de lleno en el campo de la estética catuliana, con el trabajo permanente de la lima en favor de un estilo literario lepidus et facetus. Sin embargo, para Séneca estas características en un estilo literario obviamente son marca de afectación (concinnitas) y esta non est ornamentum virile (es decir, el estilo ideal, para Séneca, está vinculado con la virilidad).

Este pasaje de Séneca confirma que, si bien el campo de la estética capilar en sentido amplio -es decir, no solo del pelo de la cabeza sino de cualquier clase de vello- se suele utilizar como un mecanismo de invectiva, generalmente de índole sexual, dentro de la literatura latina -como veremos infra en los ejemplos de Lucilio, Catulo, Cicerón, los Carmina Priapea, Petronio, Séneca y Marcial-, hay casos en donde la terminología asociada con lo hirsuto puede implicar una referencia ulterior a la propia literatura, es decir, puede ser utilizada como programática y metapoética. Como afirma Sandy (1971, p. 57), hablando del c. 16:

First, there are only a few explicit statements of literary principles in Catullus (...). Second, we generally have to rely on inference to determine his ars poetica; about all this process tells us is that, as in poem 16 , Catullus commends his nugae for their lepos, sal, risus, ioci, venustas, and so on. Finally, a mixture of a particular occasion or situation with literary doctrine seems to be a feature peculiar to Catullus. (...) Many of Catullus' practices and declarations are truly revolutionary. Poem 16 certainly is.

11. "Conoces a esos jóvenes afeminados, relucientes por su barba y cabellera, todos ellos como salidos de una caja: no esperes de ellos energía, ni solidez alguna. El estilo es el adorno del alma: si es pulido, acicalado y artificial, muestra que aquella es también insincera y se halla algo debilitada. Porque la afectación no es ornamento varonil”. Trad. de Roca Meliá (1989). 


\section{Pilosidad, vellosidad y depilación en Roma: algunos testimonios}

En este punto, veremos la cuestión de la propia terminología latina referida a la pilosidad y sus implicancias, es decir, qué significaba para los romanos una descripción de o una alusión a alguien "peludo", "barbudo", "depilado", etc. Para dar cuenta de este asunto, comenzaremos con algunas reflexiones culturales generales de la crítica sobre este problema en la Antigüedad, para luego revisar los testimonios de la literatura latina.

Boswell (1980) afirma que existe una extrema inconsistencia en muchos escritores acerca de qué elementos vuelven evidentemente afeminado a un varón, por lo que hace que sus testimonios carezcan de un valor sistemático. Por ejemplo, Marcial, en numerosos epigramas, critica la belleza y la fealdad que le parecen "afeminadas", la apariencia desaliñada y la vanidad, el olor corporal y el aroma perfumado, la depilación y la vellosidad, las mejillas lisas y la barba; en resumen, cualquier característica que le desagrade, todo puede ser "afeminado" dependiendo de su extrema subjetividad. Por el lado del estoicismo, se encuentra la censura del afeitado y del cuerpo depilado; quienes lo practican son calificados como "antinaturales" " "afeminados" (cfr. Sen. Rh., Controv. I, praef. 10; Epict. 3.1.28 fr.; Sen., QNat. 7.31.2, Ep. 47, 122; Aul. Gell. 3.6), pero ningún pensador estoico sugiere que fuera algo característico de los varones homosexuales (Boswell, 1980, p. 77, n. 72).

Por su parte, Gleason (1995) afirma que en épocas de Clemente de Alejandría (ca.150-ca.215) la barba se consideraba la marca distintiva de un hombre, ya que la vellosidad en general es la prueba de una naturaleza varonil. Es la misma idea de Epicteto (Diatr.1.16.11), quien ve en las características sexuales secundarias "signos" que funcionan lingüísticamente: anuncian "desde lejos" que alguien es un varón. Asimismo, Aristóteles (Gen. an. 765b, 783b) afirmaba que el pelo natural del hombre era el producto de la misma abundancia de calor interior que producía su esperma. La depilación, por tanto, podía considerarse una práctica especialmente peligrosa. Era una forma de "ablandarse”, de entregarse al placer de la pasividad. Y dentro del estoicismo, como se dijo, el tema del pelo adquirió una significación simbólica, ya que la masculinidad era definida de este modo por la propia naturaleza. Así, Gleason (1995, p. 69) cita a Musonio, que alababa la barba como "un símbolo del varón, como la cresta del gallo y la melena del león” (fr. 21), y a Epicteto, que le aconsejó a un joven estudiante de retórica, bien peinado y depilado: "Deja la cabellera a Quien te la modeló como Él quiso" (Diatr. 3.1.26). Así las cosas, la naturaleza hizo a las mujeres suaves y a los hombres hirsutos. El problema surge cuando vemos a un hombre sin pelo: si es algo de nacimiento, si nace lampiño, era visto como un fenómeno, un signo ominoso. Pero es peor si un hombre se depila: según Epicteto (Diatr. 3.1.31), quien se depile debería eliminar la causa del crecimiento de su pelo para no engañar a los demás y convertirse enteramente en una mujer. Clemente de Alejandría (Paed.3.3.20) no solo veía la depilación como algo impío, contra natura, sino también ridículo: subraya la indignidad de las posturas que deben adoptar quienes se depilan en público, retorciéndose para tapar sus genitales y en posiciones absolutamente indecorosas.

Sin embargo, a pesar de estas afirmaciones sobre el pelo y la vellosidad, podemos decir que "no todo el que es peludo es macho", dado que también el hecho de mostrarse velludo podía ser visto como una pose. Además, es importante recordar que las críticas de los moralistas hirsutos no contaban con 
un asentimiento universal. Si alguien se mostraba depilado, no necesariamente quería ser reconocido como sexualmente pasivo, sino que también podía entenderse como sinónimo de elegancia. Según Gleason (1995, p. 74), "these habits, while they might in some circumstances constitute a shorthand key to their practitioner's sexual preferences, might also bear a more generalized penumbra of meaning and indicate nothing more than his aspirations to elegance". En este sentido, parece haber habido límites vinculados con la "etiqueta": no se podía ir a un acto cívico ni a una cena con el atuendo desaliñado de un filósofo cínico; Séneca (Ep.114.14) decía que cierto refinamiento era necesario: por ejemplo, se podían depilar las axilas, pero nunca las piernas. Tal como afirma Gleason (1995, p. 75, n. 90), el contexto de esta afirmación en dicha epístola es el de crítica literaria: ${ }^{12}$ la depilación no es más que el vehículo de una metáfora sobre el estilo oratorio, un hecho que refuerza la afirmación de que en la antigüedad los juicios sobre el habla estaban sexualizados porque el habla era una variable esencial en la construcción social de la masculinidad, como ocurre en Catulo.

En definitiva, la falta de testimonios de los defensores del "estilo suave" dificulta nuestra apreciación de la variedad de actitudes ante la depilación y lo que verdaderamente se pensaba sobre los pilosi. Gleason (1995, p. 76) afirma que la postura de los romanos hacia el afeminamiento y la depilación era muy variada, y está claro que algunos segmentos de la sociedad de clase alta aceptaban más estas prácticas que otros. La causa probable puede deberse a "the constraints on the sort of masculine persona that people felt entitled to adopt in writing". La depilación parece haber sido un tema que un autor no podía tratar por escrito sin dispositivos de distanciamiento, "among which the brine of invective appears to have been preferred for its sterilizing effect". Indudablemente, el sistema represivo de la cultura romana se imponía a través de la amenaza de la censura y el ridículo, y la apariencia de un varón adulto no escapaba en absoluto de la mirada inquisidora del otro. Ser elegante seguramente debió haber conllevado el peligro permanente de ser señalado con el dedo y ridiculizado bajo el rótulo denigrante de "afeminado".

En cuanto a las ocurrencias de pilosus en el ThLL (10.1.2142.27-46), referidas a seres humanos, ${ }^{13}$ implican en la mayoría de los casos una connotación sexual y/o con un sentido casi siempre peyorativo para el sujeto implicado:

a) Lucil. 73 in bulgam penetrare pilosam ("penetrar un morral peludo"), cfr. CIL 4.1830 add. p. 212, futuitur cunnus [pil]ossus multo melius [qu]am glaber; / e[ad]em continet uaporem et eadem u[err] it mentulam ("es mucho mejor metérsela a una concha peluda que a una pelada; conserva el calor y estimula la verga”). Si bien no tenemos el contexto del pasaje de Lucilio, es muy probable que esté describiendo un acto sexual, con un sentido similar al de la cita del CIL. Adams (1982, p. 76) sostiene que en latín clásico existía una distinción entre capillus, el pelo de la cabeza, y pilus, el pelo del cuerpo, que incluía el vello púbico, aunque en algunos casos esa distinción se hace difusa (cfr. Petron. 109.9, Sen. Dial. 9.8.3, Plin. Nat.11.130).

b) Cic. Pis. 1 non ... nos color iste servilis, non pilosae genae, non dentes putridae Pisonis deceperunt ("no nos engañaron ni ese color servil, ni las mejillas hirsutas, ni los dientes podridos de Pisón”).

12. Como ocurre también en la ya analizada Ep. 115.

13. Citamos solo testimonios de literatura pagana. 
Cicerón se refiere a Lucio Calpurnio Pisón con un retrato en el que sobresale el aspecto tosco y vulgar, con el que el orador intenta influir negativamente en el auditorio.

c) Nuestro verso catuliano.

d) Catull. 33.7 natis pilosas, fili cinaede, non potes asse venditare. Será analizado infra.

e) Scrib. Larg. 230 palpebram pilosam ("párpado hirsuto"). Se trata de un texto médico en donde se receta la cura para estos casos.

f) Mart. 9.27.7 (ad pathicum depilatum): citamos los primeros siete versos:

Cum depilatos, Chreste, coleos portes

Et uulturino mentulam parem collo

Et prostitutis leuius caput culis,

Nec uiuat ullus in tuo pilus crure,

Purgentque saeuae cana labra uolsellae,

Curios, Camillos, Quintios, Numas, Ancos,

Et quidquid umquam legimus pilosorum

Loqueris...

Aunque tengás depiladas las bolas, Cresto, y la pija igual que el cuello de un buitre, y la cabeza más suave que los culos prostituidos, y aunque no haya ni un pelo en tus piernas y las pinzas pinchen crueles tu bigote canoso, hablás de los Curios, de los Camilos, de los Quincios, de los Numas, de los Ancos y de todos los hombres de pelo en pecho que alguna vez leímos.

El ataque a Cresto se basa, en buena medida, en su carácter de pathicus depilatus, por lo que el vello es un tema central del epigrama.

g) Mart. 9.47.5 (ad quendam barba philosophos imitantem)

Democritos, Zenonas inexplicitosque Platonas

Quidquid et hirsutis squalet imaginibus,

Sic quasi Pythagorae loqueris successor et heres.

Praependet sane nec tibi barba minor:

Sed, quod et hircosis carum est et turpe pilosis,

In molli rigidam clune libenter habes.

$\mathrm{Tu}$, qui sectarum causas et pondera nosti,

Dic mihi, percidi, Pannyche, dogma quod est?

A los Demócritos, a los Zenones y Platones, que no has leído en tu puta vida, y a todos aquellos que se representan desaliñados en bustos hirsutos, los mencionás como si fueras el sucesor y heredero de Pitágoras. Y, ciertamente, no te cuelga una barba menos tupida. Pero, algo que para los hediondos es apreciado y vergonzoso para los de pelo en pecho, a vos te gusta tenerla dura entre tus suaves nalgas. Vos que conocés los orígenes y el peso de las escuelas filosóficas, decime, Pánico, ¿qué principio filosófico es que te la pongan? 
La diatriba contra Pánico tiene tres momentos en donde la pilosidad es protagonista, siempre relacionada con los filósofos y su aspecto desaliñado, del que Pánico es una triste imitación.

h) Mart.12.59.4 pilosus ... colonus. El epigrama trata de los besadores inoportunos e insoportables que reciben a quien vuelve a Roma después de un largo viaje. Dentro de la lista de estos impertinentes, aparece un pilosus colonus con unas características particulares: te pilosus / Hircoso premit osculo colonus ("a vos te da un beso con gusto a chivo un colono peludo"). ${ }^{14}$

i) Priap. 46.4 puella ... ursis asperior pilosiorque ("una chica... más hirsuta y peluda que los osos”): se trata de una chica poco atractiva para tener sexo. Si tomamos la referencia del CIL 4.1830 (cfr. a), es evidente que acá se enfatiza que la vellosidad de la chica era excesiva.

j) Juv. 14.194 naris ... pilosas. Los versos son estos: Juv. 14.194-195 sed caput intactum buxo narisque pilosas / adnotet et grandes miretur Laelius alas ("que Lelio se dé cuenta de que el peine no tocó tu cabeza y de tu nariz hirsuta y admire tus grandes hombros"). Se trata de las enseñanzas de un padre moderno a su hijo, que lo educa en la ambición y en la avaricia, opuestas al modelo del mos maiorum. La enseñanza incluye una alusión a la pilosidad como modelo de una virtud, en este caso, simulada, como ocurre en Juv. 2.11-13, con otros términos.

\section{La pilosidad en Catulo: terminología y ocurrencias}

Como se dijo supra, la otra ocurrencia del término pilosus en Catulo aparece en el c. 33 (cfr. 3, d). Se trata de un poema en forma de invectiva contra un tal Vibenio y su hijo, quienes roban ropas en los baños públicos. El término pilosus aparece asociado, nuevamente, con una temática sexual, en este caso para describir al hijo de Vibenio, que es acusado por Catulo de ser un cinaedus $^{15}$ (vv. 5-8):

cur non exilium malasque in oras
itis, quandoquidem patris rapinae
notae sunt populo, et natis pilosas,
fili, non potes asse uenditare?

¿por qué no se van exiliados hacia unas costas malditas, ya que todo el pueblo conoce los robos del padre y vos, el hijo, no podés vender ni por un as tu culo peludo?.

Catulo refiere que el hijo de Vibenio ya ha atravesado la edad del efebo, cuando el varón tiene la piel lisa y lampiña, y cuando seguramente le daba rédito económico al padre -hay una alusión en el poema a la prostitución-. Ahora, ni siquiera a precio de oferta consigue clientes. En este caso, el término pilosus acompaña a un sustantivo que, como en el c. 16, alude a un contexto sexual, con la diferencia de que aquí el protagonista es un hombre joven, un cinaedus, que ha perdido sus encantos

14. En otros epigramas, Marcial toca el tema de la vellosidad relacionado con la sexualidad, pero no utiliza pilosus sino pilus, como en 6.56 .

15. Seguramente por esto Fordyce (1961) tampoco lo comenta. 
como prostituto, mientras que Furio y Aurelio son hombres ya adultos, cuya potencia sexual está muy reducida (a tal punto que solo los versos de Catulo funcionan como un vigorizante), pero que no pueden ser definidos como pathici. Vemos, entonces, cómo el término pilosus puede ser aplicado tanto a un cinaedus como a quienes no lo son. Sin embargo, en el c. 33 pilosus no puede ser interpretado metapoéticamente, simplemente, porque el carmen en general no tiene ningún tipo de implicancia programática.

El campo semántico de la pilosidad reaparece en el c. 37, pero bajo otro término: capillatus. En este carmen, Lesbia se ve retratada como una meretrix de una taberna de mala fama (salax taberna). Catulo, indignado con los vividores que pululan por la taberna, presta especial atención a un tal Egnatius. Transcribimos los versos finales del poema (vv. 14-20):

\author{
Hanc boni beatique \\ omnes amatis, et quidem, quod indignum est, \\ omnes pusilli et semitarii moechi; \\ tu praeter omnes une de capillatis, \\ cuniculosae Celtiberiae fili, \\ Egnati, opaca quem bonum facit barba \\ et dens Hibera defricatus urina.
}

Todos ustedes, nobles y ricos señores, le hacen el amor [a Lesbia] y, en verdad, lo peor de todo es que son todos unos pobres diablos y putañeros de callejón, especialmente vos, Egnacio, uno de los melenudos, hijo de la conejera Celtiberia, con esa barba oscura que te hace un ganador y esos dientes, que brillan por la meada ibérica.

La interpretación de Krostenko (2001, p. 267) es atendible: capillati se refiere a los jóvenes que llevaban el pelo largo para poder hacerse peinados a la moda, una suerte de galanes. Sin embargo, la descripción de Catulo pone énfasis en la afectación del muchacho, que convierte esa elegancia en algo despreciable, vinculado con lo extranjero y, por lo tanto, lo vulgar, lo prosaico. E1 hecho de que aparezca la expresión cuniculosae Celtiberiae define todo, ya que, como sabemos por el c. 25, no hay nada más suave que el pelo de un conejo español; así, la mollitia de Egnacio se debe no a su "understanding of the semiotics of contemporary Roman culture but to his origins in Celtiberia, the land of soft bunnies" (Krostenko, 2001, p. 268). Egnacio sufre de provincialismo: carece absolutamente de urbanitas. Así, nuevamente, para Catulo el elemento "capilar" tiene connotaciones negativas y burlonas: si en el $c$. 16 la burla se dirigía hacia unos adultos impotentes, aquí se trata de un típico elemento xenófobo romano. Hay otro texto donde llevar el pelo largo también es una marca de ambigüedad: por un lado, es sinónimo de atractivo sexual, pero, por el otro, nuevamente, el problema es quiénes se sienten atraídos por este tipo de cualidad física. En el Satyricon, Trimalción se presenta como un senex calvus que juega a la pelota entre pueros capillatos (Petron. 27.1); luego, en una écfrasis de una suerte de mural autobiográfico del liberto, Encolpio nos cuenta que ipse Trimalchio capillatus caduceum tenebat (Petron. 29.3, "el mismísimo Trimalción tenía un caduceo"); finalmente, en el cuento de Nicerote del hombrelobo, Trimalción introduce el relato así: cum adhuc capillatus essem (Petron. 63.3, "cuando todavía tenía el pelo largo"). Estas descripciones demuestran que para Trimalción es una tragedia ser calvo, ya que recuerda su larga cabellera con mucha nostalgia. Pero, más importante, Petronio expone el contraste 
entre la calvicie del liberto y la cabellera de sus esclavos, pueri delicati, como un modo de marcar que esos esclavos eran muy deseables sexualmente. Esto podría implicar un aspecto positivo de capillatus: sin embargo, la habitual ironía petroniana nos señala que un capillatus era un cinaedus apetecible por un personaje ignorante, burdo, excéntrico y extranjero como Trimalción. Es decir, parece haber una distinción entre capillatus y pilosus: mientras que el primero puede dar lugar a este tipo particular de ambigüedad, el segundo siempre implica algo negativo.

En la sección de los licenciosos fescennini versus (vv. 121-155) del c. 61 (el epitalamio para las bodas de L. Manlio Torcuato y Junia Arunculeya), durante la procesión nupcial, se alude a los gustos sexuales del novio en su vida de soltero con algunas menciones a lo capilar (vv. 129-136):

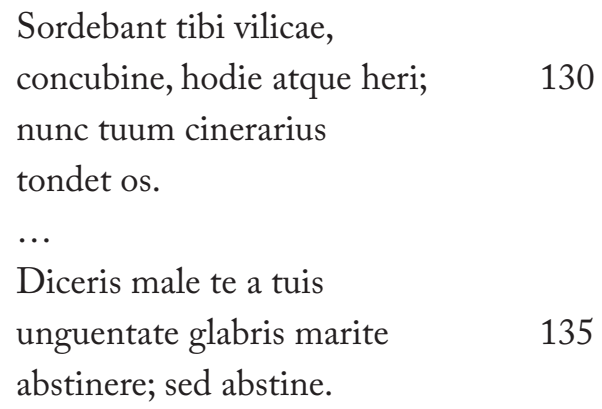

Te daban asco las campesinas, concubino, ayer y aun hoy; ahora el peluquero va a cortarte el pelo. (...) Andan diciendo, marido perfumado, que renunciás de mala gana a tus chicos recién depilados; pero renunciá.

Nuevamente, un puer delicatus (en este caso, el concubino del ahora esposo) debe ir al peluquero para que este le corte el pelo. El verbo tondere aquí es ambiguo porque puede significar "rapar", "cortar el pelo" y "afeitar la barba”. El canto epitalámico es hiriente contra el concubinus, que despreciaba a las vilicae: ahora, debe enfrentarse con su peor enemigo, el peluquero. Pero la sutileza de Catulo, que define toda la escena, radica en que el peluquero, en general, se denomina tonsor, y aquí se lo llama cinerarius: habitualmente, este era el encargado de rizar el largo cabello del concubinus, al calentar en las cenizas (cfr. cinis) los hierros para hacer los rulos; ${ }^{16}$ ahora, ya está demasiado viejo (como lo revela la posible referencia a la barba) y los rulos dejarán lugar a un cruel corte de pelo. En la siguiente estrofa, el coro canta que el novio comparte con el concubinus algunos rasgos a los que debe renunciar, como estar perfumado (unguentate) o como acostarse con sus esclavos depilados (tuis... glabris). Estos gustos de soltero pueden poner en peligro el papel sexual que ahora debe desempeñar como marido. En este poema, entonces, puede apreciarse que el motivo de la capilaridad está vinculado con los cinaedi desde una perspectiva burlona, típica de los versos fesceninos, pero que no tienen una implicancia metaliteraria.

En definitiva, a lo largo de este artículo, hemos recorrido múltiples aspectos vinculados con la expresión his pilosis del v. 10 del c. 16 de Catulo. Como vimos en la primera sección, muchos

16. Cfr. Fordyce (1961, p. 249). 
críticos coinciden en que el poema puede ser leído de un modo programático, pero ninguno alude a la importancia de his pilosis en esta lectura. Aunque hemos encontrado que lo habitual dentro de la literatura latina es que la pilosidad (entendida en sus diversas manifestaciones) es un arma muy efectiva para poner en práctica un bullying punzante contra los acusados de ser pathici, hay tres pasajes que nos advierten que es posible leer el campo de la "estética capilar hirsuta" de manera metapoética: el testimonio de Séneca en su Epistula 115 y dos poemas de Catulo (c. 16 y 37). En el segundo, esta posibilidad está implícita en el tratamiento de Egnatius, un capillatus hispano y, por lo tanto, extranjero y rusticus, alejado de la elegancia estilística de un poeta novus: para Catulo, Egnatius es absolutamente indigno de acostarse con la delicada Lesbia. Sin embargo, es el c. 16 el que muestra con mayor énfasis la duplicidad de sentidos de pilosus: de por sí, el término no tiene una connotación positiva, y en el poema presenta una clara connotación sexual, porque Furio y Aurelio, los pilosi, ya no pueden movere lumbos, es decir, tener sexo, porque son adultos impotentes. Pilosi, en este contexto, hace referencia muy posiblemente a la pilosidad que es común con el paso de los años: puede aludir no solo a la vellosidad en zonas estéticamente odiosas, como la espalda, sino también a los pelos hirsutos en las orejas o en las fosas nasales. Este primer sentido de pilosus es evidente en el carmen. Sin embargo, también hay otro sentido, que es el que hemos intentado definir en nuestro trabajo: his pilosis se refiere metapoéticamente a aquellos incapaces de entender los delicados y graciosos versos catulianos, a aquellos que creyeron que sus carmina eran afeminados y, por lo tanto, hacían de Catulo un pathicus. Si Furio y Aurelio hubieran sido buenos lectores, si hubieran sido sofisticados y no vulgares pilosi, podrían haber entendido que los versos catulianos tienen sal y lepos, que son molliculi y parum pudici justamente para poder excitarlos a ellos, pobres impotentes. Como señalaron varios críticos, las duplicidades del lenguaje de Catulo implican que los términos deban ser estudiados detenidamente en cada contexto: esa sutileza es demasiado pedir para estos pilosi, cuya rusticidad exegética es tan notable que se hace evidente en su desagradable pilosidad.

\section{Referencias bibliográficas}

Adams, J. N. (1982). The Latin Sexual Vocabulary. London, Duckworth.

Arkins, B. (1982). Sexuality in Catullus. Hildesheim-Zurich-New York, Olms.

Baehrens, E. (1885). Catulli Veronensis liber. Lipsiae, Teubner.

Batstone, W. (1998). Dry Pumice and the Programmatic Language of Catullus 1. Classical Philology, 93, 125-135.

Batstone, W. (2007). Catullus and the Programmatic Poem: The Origins, Scope, and Utility of a Concept. En Skinner, M. (comp.), A Companion to Catullus (pp. 235-253). Malden, Blackwell.

Boswell, J. (1980). Christianity, Social Tolerance, and Homosexuality Gay People in Western Europe from the Beginning of the Christian Era to the Fourteenth Century. London, The University of Chicago Press.

Buchheit, V. (1976). Sal et lepos versiculorum (Cattul C. 16). Hermes, 104, 331-347. 
Cantarella, E. (1988). Secondo natura. La bisessualità nel mondo antico. Roma, Editori Riuniti.

Della Corte, F. (1977). Catullo, le Poesie. Milano, Fondazione Lorenzo Valla \& Arnaldo Mondadori.

Fitzgerald, W. (1995). Catullan Provocations: Lyric Poetry and the Drama of Position. Berkeley, Los Angeles and London, University of California Press.

Fordyce, E. J. (1961). Catullus: A Commentary. Oxford, Oxford University Press.

Gleason, M. (1995). Making Men Sophists and Self-presentation in Ancient Rome. Princeton, Princeton University Press.

Herzfeld, M. (1985). The Poetics of Manhood: Contest and Identity in a Cretan Mountain Village. Princeton, Princeton University Press.

Kinsey, T. E. (1966). Catullus 16. Latomus, 25, 101-106.

Kroll, W. (1923). C. Valerius Catullus, herausg. und erklärt. Leipzig, Teubner.

Krostenko, B. (2001). “Arbitria Vrbanitatis”: Language, Style, and Characterization in Catullus C. 39 and 37. Classical Antiquity, 20, 239-272.

Krostenko, B. (2007). Catullus and Elite Republican Social Discourse. En Skinner, M. (comp.), $A$ Companion to Catullus (pp. 212-232). Malden, Blackwell.

Lateiner, D. (1977). Obscenity in Catullus. Ramus, 6, 15-32.

Lenchantin de Gubernatis, M. (1932). Il libro di Catullo Veronese; introduzione testo e commento. Chiantore, Torino.

Manwell, E. (2007). Gender and Masculinity. En Skinner, M. (comp.), A Companion to Catullus (pp. 111-128). Malden, Blackwell.

Marrón, G. (2012). Habeas Corpus. Latin, sexo y traducción. Bahía Blanca, VOX.

Mynors, R. (1957). C. Valerii Catulli Carmina. Oxford, Oxford University Press.

Rankin, H. D. (1976). Poem 16 of Catullus. Symbolae Osloenses, 51, 87-94.

Richardson, L. (1963). Furi et Aureli, Comites Catulli. CPh, 58, 93-106.

Richlin, A. (2007). Catullus and the Art of Crudity. En Gaisser,J. (comp.), Oxford Readings in Classical Studies. Catullus (pp. 282-302). Oxford, Oxford University Press. (Original de 1992.)

Roca Meliá, I. (1989). Séneca. Epistolas morales a Lucilio II (Epistolas 81-125). Madrid, Gredos.

Sandy, G. (1971). Catullus 16. Phoenix, 25, 51-57.

Selden, D. (2007). "Ceveat lector": Catullus and the Rhetoric of Performance. En Gaisser, J. (comp.), Oxford Readings in Classical Studies. Catullus (pp. 490-559). Oxford, Oxford University Press.

Schievenin, R. (2000). Poesia e turpiloquio nel carme 16 di Catullo. MD, 44, 195-209. 
Skinner, M. (2003). Catullus in Verona: A Reading of the Elegiac Libellus, Poems 65-116. Ohio, Ohio State University Press.

Skinner, M. (comp.) (2007). A Companion to Catullus. Malden, Blackwell.

Thomson, D. F. S. (1997). Catullus. Edited with a Textual and Interpretative Commentary. Toronto, University of Toronto Press.

Wray, D. (2001). Catullus and the Poetics of Roman Manhood. Cambridge, Cambridge University Press.

*Marcos Carmignani es Doctor en Letras, Licenciado en Letras Modernas y Licenciado en Letras Clásicas por la Universidad Nacional de Córdoba. Se desempeña como Profesor Titular de Filología Latina y Adjunto a cargo de Historia de la Literatura Latina en la UNC. Es Investigador del CONICET y editor general de la revista Ordia Prima. Sus áreas de investigación son la novela romana, los centones virgilianos, la teoría intertextual y la tradición clásica en la obra de Borges. Algunos de sus trabajos pueden encontrarse en: https://conicet-ar.academia.edu/MarcosCarmignani.

RECIBIDO: 06/04/2021

ACEPTADO: 04/06/2021 